\title{
Great ideas for teaching optics
}

\section{Stephen Pompea, Larry Stepp}

Stephen M. Pompea, Larry M. Stepp, "Great ideas for teaching optics," Proc. SPIE 2525, 1995 International Conference on Education in Optics, (13 October 1995); doi: 10.1117/12.224009

SPIE Event: SPIE's 1995 International Symposium on Optical Science, SPIE. Engineering, and Instrumentation, 1995, San Diego, CA, United States 


\title{
Great ideas for teaching optics
}

\author{
Stephen M. Pompea ${ }^{1}$ \\ Pompea \& Associates \\ 1321 East Tenth Street \\ Tucson, Arizona 85719-5808 \\ Electronic mail: spompea@as.arizona.edu \\ Larry Stepp \\ Gemini 8-m Telescopes Project \\ P. O. Box 26732 \\ Tucson, Arizona 85726 \\ Electronic mail lstepp@gemini.edu
}

\begin{abstract}
There are many excellent science teachers, each of whom has a number of well-tested, successful demonstrations or ideas on how to teach some aspect of the field of optics. This reservoir of ideas is a valuable resource for integrating optics teaching into other science disciplines and into the general curriculum. How do we tap this resource? This paper outlines the usefulness of collecting ideas on optics teaching with the intention of publishing the collection in book form, while giving credit to each contributor. The authors are currently involved in collecting a wide range of ideas on teaching optics in order to create a book that is useful in a variety of teaching situations. We hope that one result of this will be to encourage the teaching of optics in a larger number of classrooms and at all grade levels.
\end{abstract}

Keywords: optics education, demonstrations, teacher training, curriculum planning

\section{INTRODUCTION}

Teaching is often a solitary profession. The teacher spends most of his or her time working with a class in an intense interaction with the content material. The more that is demanded from the students in the way of writing and examinations, the greater the teacher's workload after class. Little time is left for the teacher to develop new materials or teaching ideas. The development and testing of new educational materials and curricula is a time-consuming process, and there is a strong need for a greater sharing of ideas among teachers. Every teacher has his or her own "great ideas" for teaching. These may be lecture starters, demonstrations, stories or anecdotes, or experiments that are particularly effective. Each teacher has developed thought or minds-on experiments designed to stimulate student thinking or to reveal misconceptions. If the "great ideas" of many different teachers could be collected into a single reference the result would be a valuable resource. The purpose of this paper is to describe the rationale for the development of a book of "Great Ideas for Teaching Optics" and to tell how teachers can contribute to such a collection.

\section{WHO SHOULD OPTICS BE TAUGHT TO?}

In the modern world everyone is affected by science. Every citizen should be aware of scientific issues and have at least a basic understanding of scientific principles. However, it can be challenging to teach science to a diverse range of students, some of whom plan to actually become scientists and many of whom do not. Each teacher assembles a repertoire of "great ideas" which often never get shared.

\footnotetext{
${ }^{1}$ Adjunct Associate Astronomer, Steward Observatory, University of Arizona 85721 Voice/Fax (520) 792-2366/622-2122
} 
These ideas are useful for teachers who:

- Want to get a lecture started in an exciting way

- Want to better be able to explain a difficult area

- Are looking for student misconceptions

- Are looking for a single guide to teaching resources

For several scientific subjects, collections of teacher's "great ideas" already exist. For example, one of the authors (SMP) was the editor of Great Ideas for Teaching Astronomy and a contributor to Great Ideas for Teaching Physics and Great Ideas for Teaching Geology (see references). These collections of ideas have proven to be useful to teachers at a variety of school levels, from grade school to college level. The interest shown by teachers in these collections is one of the prime motivations for a more specialized collection in optics.

Optics is often thought of as a senior-level or graduate-level class. Our starting point for this collection is the premise that there is no one grade level where optics should be taught, particularly since optics is one of the most demonstrable of all sciences. We believe that optics can be taught in different ways at all grade levels. We also strongly believe that students must have an experience of optics and optical phenomena before they can learn fundamental concepts, and that they should be given an opportunity to "play" with optics. Just as physics concepts such as acceleration may be meaningless to students until they have experienced the phenomena of acceleration, so too optics concepts such as polarization must be demonstrated and experienced before teaching in this area can be particularly effective.

To many people, a pair of binoculars or a camera are magic, partly because these people have never seen the interior and cannot name any of the parts, or how they work together. A curiosity about how things work is at the start of a study of science and engineering. Almost every person working in science can relate some story of an early fascination with some object or phenomena.

Optical phenomena can be explored at any grade level. Primary level students should be exposed to as many optical phenomena as possible in order to help develop a way of seeing. Just as a fourth grade lesson on clouds, which differentiates the different types of clouds, allows students to understand and be more aware of the sky, so too can an exposure to optical phenomena enhances the ability of students to develop an appreciation and understanding of optics. Simple lessons on shadows, swimming pool optics, or how binoculars work can be invaluable in training young minds to see and understand the principles of optics. In this paper, we use the word optics in the fullest definition imaginable. We are trying to explore how any aspect of light or electromagnetic radiation or its detection can be taught or be of interest to students.

\section{TOPICS FOR OPTICS TEACHING}

What topics in optics might be appropriate for this collection? Our desire is to include a wide range of ideas on as many optics topics as possible. Since optics will often be taught as part of another course (perhaps not even as part of a science course), there may be as much interest in an idea that professional optical scientists might consider a curiosity as an area that is more in the mainstream.

For example, an idea that relates the history and limitations of eyeglasses may have great general value even though it is not a topic that would be discussed in an optics textbook. Similarly a discussion of diseases that affect the eyes may have a wide application to teachers in a number of areas. Of course we want a good representation of the material in the standard college optics books as well. We do not want to limit in any way teaching ideas that are appropriate for the most advanced students. However, we do want to cover the field in as broad a manner as possible and to include ideas that will be of interest to novices in the field, even if they are obvious to professionals. 


\section{Some Possible Topics}

- Working with Students

- Electromagnetic Waves

- Shadows, Eclipses

- Pinhole Cameras

- Propagation of Light: Reflection

- Propagation of Light: Refraction:

- Fiber Optics, Total Internal Reflection

- Dispersion Phenomena

- Atmospheric Phenomena

- Geometrical Optics, Aberrations

- Cameras and Photography

- Film

- Human Eye

- Visual Processing

- Color

- Diffraction

- Energy concentrators

- Light sources and detectors

- Favorite books, movies, computer programs for teaching optics
- Microscopes and Telescopes

- Quantum Nature of Light

- Lasers

- Formation of Images

- Holography

- Misconceptions about Optics

- Ocean Optics

- Polarization: Polarizers, Dichroism

- Birefringence

- Scattering and Polarization

- Interference and Interferometers

- Fourier Optics

- Optics in Literature

- Optics in Films

- Humor and Optics

- Optics in Entertainment

- Fluorescence

- Optical Coatings

- Sources for inexpensive materials for experiments

\section{APPROPRIATE TYPES OF TEACHING IDEAS}

There are many types of teaching ideas that may be appropriate for this collection. Here are a few categories:

1. LABORATORY EXPERIMENTS: These could include a brief recipe or description of a lab experiment. For example, one experiment could involve freezing water in an appropriately shaped dish to make an ice lens. Another example of an interesting laboratory experiment is described in the following quotation from reference 2:

\section{Optical Effects of Spherical Lenses}

In studying the ray optics of lenses, the students learn how to manipulate the lens equation to predict the magnification and location of images from lenses of various shapes and index of refraction. But seeing is believing.

An effective demonstration of the effect of the surrounding medium and the index of refraction of the lens on the size and location of the image can be shown with a hollow glass sphere and a tank of water. The glass sphere can be one made from a flask in chemistry lab or a container jar for flowers, for example. It should be one that can be stoppered to be air- and water-tight. If an object such as a bright red ball is placed in the container and lowered into the tank of water, the image seen will appear smaller in size than in real life. Conversely, if the ball is placed in the container filled with water, the image seen will be larger than in real life. Of course if neither the tank nor container has water in them the image will be the same as in real life.

One can also experiment with using alcohol or glycerine as the medium in the container and see the effect on the image when placed in the tank of water. --James E. Kettler, Ohio University 
2. Demonstrations: These could be any kind of demonstration appropriate for the whole class to watch or experience. An example of this is the use of milk particles in a beaker of water on an overhead projector to illustrate why the sky is blue. The following quotation from reference 3 describes another example of a good classroom demonstration:

\section{How the Thin-Section Technique Works}

To demonstrate how the thin-section technique works, obtain two large (10 cm or more square) polarizing filters. These are available at low cost from most science supply houses. Also get a large (at least $5 \mathrm{~cm}$ in diameter and about $1 / 2 \mathrm{~mm}$ thick) sheet of muscovite mica. It need not be a perfectly smooth cleavage sheet; in fact, it works better if it isn't, but it must be transparent. Put the mica inside a page protector, trim to taste, then seal the edges with tape. This will protect the sample from wear and tear.

Place one filter on the overhead projector and note that the light is somewhat dimmed, but nothing else seems to happen. Place the second polarizer atop the first, with polarization directions parallel. Note that there is a little more dimming, but nothing else. Now rotate the top polarizer $90^{\circ}$ to demonstrate extinction. This in itself will astonish many students.

One might intuitively expect that, if we put the mica between the polarizers, no light will come through. Instead, if the mica is put between the polarizers with the right orientation, bright colors appear. They show up quite well on the projection screen. For introductory classes, I point out that the crystal structure of the mineral affects the polarized light, and that we can use polarized light as a simple but powerful probe of the atomic structure of minerals. For more advanced classes, you can demonstrate extinction and other effects.

What happens if we put the mica between parallel polarizers? Intuition might suggest we will see just what we see in ordinary light, but again intuition is wrong. A different set of interference colors appears. Parallel polarizers are used much more rarely than crossed polarizers, but interference colors occur in this case as well. ---Steven I. Dutch, University of Wisconsin--Green Bay

3. EXPERIMENTS FOR STUDENTS To Do INDIVIDUALlY: These are activities that can be done by students without much supervision. Students would individually (or in small groups) demonstrate or build something. An example of this category is an experiment with two pieces of polarizing film and a crystal of calcite. Another example of an experiment that students can do individually is related in the following quotation from reference 1 :

\section{A Demonstration of the Resolving Power of a Telescope}

To demonstrate graphically that resolving power depends on the aperture of a telescope, prepare a card with a narrow slot that is a mm or two wide, and as long as the aperture of the telescope. (This also can be done with two cards and a pair of binoculars.) Fix the card in front of the telescope or binocular objective(s) with the slot(s) vertical, and have the students look at a brick wall in the middle distance, such that individual bricks are visible. They should be able to see the horizontal mortar joints but not the vertical ones. Then place the cards such that the slots are horizontal: the vertical joints should now appear, and the horizontal ones disappear. More advanced students can relate what they see to the spatial frequencies passed by the slot in the two orthogonal directions. ---William G. Weller, A.U.R.A.

4. Puzzlers and Thought Experiments: These could be used in a variety of teaching situations. An example of this kind of idea would be to give students a drawing of the cross sectional of a fresnel lens and ask them to predict how it would affect a plane wavefront. Another example of a thought experiment is to ask students how the properties of a lens would change if a pie shaped area of the lens was removed or if the lens was cut in half and only one piece was used.

5. Misconceptions Aвоut Optics: Misconceptions about optics are good starting points for discussions and for an understanding of prior correct or incorrect knowledge. An example of a misconception is that all lasers are red in color. 
6. TRICKS OR MNEMONIC DEVICES: These can help students understand or remember material.

The ideas or demonstrations need not be complex. Complex ideas may be broken into smaller units. As in the examples quoted above, each idea should be written as a few paragraphs succinctly describing the idea in a form that will be immediately useful to a teacher. Each idea will have a title and will be placed in a chapter based on the subject matter. So a chapter on polarization, for example, will have thought experiments, demonstrations, anecdotes, etc. about polarization.

Be sure to include any extension activities that can be used to extend the principles or lessons exhibited in a demonstration. Almost any type of idea is worthy of consideration for this collection. However, a few classes of ideas will probably not be selected for inclusion. For example, experiments or demonstrations requiring long detailed procedures will probably not be chosen because of their length. Since many successful teaching ideas are only a few paragraphs in length, longer ideas will only be used when they are worth the value of several shorter ideas. Enough detail should be given to ensure a successful outcome, though. Also, ideas with excessive reliance on complicated equipment will have less of a chance of being chosen, because the equipment for such a demonstration may not be available in most schools or even colleges.

\section{CONCLUSION}

We believe that all students can benefit from experience with optics and optical phenomena. Most students will never take an "optics" course per se, but optical phenomena and optical principles can be taught as part of many other courses such as anatomy, astronomy, biology, chemistry, geology, physics, psychology, etc. These optical phenomena can be explored at any grade level provided the teacher is well-prepared with appropriate demonstrations, experiments, and thought-provoking questions.

There are many excellent science teachers, each of whom has a number of well-tested, successful ideas on how to teach some aspect of the field of optics. We believe that if a number of people who work in the field of optics or who teach about optical phenomena contributed their best ideas to a collection, the result would be a very useful resource.

To help provide ideas for teachers at all levels from primary school to college, the authors are putting together a collection of "great ideas for teaching optics" in a form that has proven successful in previous books for teaching astronomy, physics, and geology. Anyone interested in contributing teaching ideas is requested to contact one of the authors at the appropriate address listed on the first page. Credit will be given to each contributor for each idea that is used.

It is our hope that the resulting book will help ensure that optics, in some form, will be taught in a larger number of classrooms over all grade levels.

\section{ACKNOWLEDGMENTS}

S. Pompea is an adjunct faculty member of Steward Observatory, University of Arizona and would like to acknowledge the university for use of its facilities. The Gemini 8-m Telescopes Project is managed by the Association of Universities for Research in Astronomy, for the National Science Foundation, under an international partnership agreement.

\section{REFERENCES}

1. S. M. Pompea, editor, Great Ideas for Teaching Astronomy, 2nd edition, West Publishing Company, St. Paul, 1994.

2. D. J. Bord and J. C. Sprott, editors, Great Ideas for Teaching Physics, West Publishing Company, St. Paul, 1991.

3. F. J. Moye and R. Wicander, editors, Great Ideas for Teaching Geology, West Publishing Company, St. Paul, 1992. 